\title{
Expression of holo-proteorhodopsin in Synechocystis sp. PCC 6803
}

Author List:

Que Chen ${ }^{1, \#}$, Jeroen B. van der Steen ${ }^{1, \#}$, Henk L. Dekker ${ }^{2}$, Srividya Ganapathy ${ }^{3}$, Willem J. de Grip ${ }^{3}$, and Klaas J. Hellingwerf ${ }^{1,4, *}$

${ }^{1}$ Molecular Microbial Physiology, Swammerdam Institute for Life Sciences, University of Amsterdam, Amsterdam, the Netherlands

${ }^{2}$ Mass Spectrometry of Biomacromolecules, Swammerdam Institute for Life Sciences, University of Amsterdam, Amsterdam, the Netherlands

${ }^{3}$ Biophysical Organic Chemistry, Leiden Institute of Chemistry, Leiden University, Leiden, the Netherlands

${ }^{4}$ BioSolar Cells, P.O. Box 98, 7600 AB, Wageningen, the Netherlands

* Corresponding author; address: P.O. Box 94232, 1090 GE Amsterdam, the Netherlands. Phone: +31

(0)20 525 7055. Fax: +31 (0)20 525 7934. Email: K.J.Hellingwerf@uva.nl.

\# These authors contributed equally to this study.

Running title: Proteorhodopsin expression in Synechocystis 


\begin{abstract}
Retinal-based photosynthesis may contribute to the free energy conversion needed for growth of an organism carrying out oxygenic photosynthesis, like a cyanobacterium. After optimization, this may even enhance the overall efficiency of phototrophic growth of such organisms in sustainability applications. As a first step towards this, we here report on functional expression of the archetype proteorhodopsin in Synechocystis sp. PCC 6803. Upon use of the moderate-strength psbA2 promoter, holo-proteorhodopsin is expressed in this cyanobacterium, at a level of up to $10^{5}$ molecules per cell, presumably in a hexameric quaternary structure, and with approximately equal distribution (on a protein-content basis) over the thylakoid and the cytoplasmic membrane fraction. These results also demonstrate that Synechocystis sp. PCC 6803 has the capacity to synthesize alltrans-retinal. Expressing a substantial amount of a heterologous opsin membrane protein causes a substantial growth retardation Synechocystis, as is clear from a strain expressing PROPS, a nonpumping mutant derivative of proteorhodopsin. Relative to this latter strain, proteorhodopsin expression, however, measurably stimulates its growth.
\end{abstract}

Keywords: proton pump, all-trans retinal, apo- and holo-protein, proton motive force, thylakoid membrane, cytoplasmic membrane

Abbreviation: DDM: $\mathrm{n}$-dodecyl- $\beta$-D-maltopyranoside; pmf: proton motive force; $\mathrm{PR}(\mathrm{s})$ : Proteorhodopsin(s); PR-His: PR with a C-terminal poly-histidine tag, PROPS: proteorhodopsin optical proton sensor; CM: cytoplasmic membrane; TM: the thylakoid membrane; SynACO: apocarotenoid15,15'-oxygenase; DCMU: 3-(3,4-dichlorophenyl)-1,1-dimethylurea; CCCP: [(3-chlorophenyl) hydrazono] malononitrile; 


\section{Introduction}

Concerns about global warming and the depletion of fossil fuels have led to an increasing need for the development of alternative, more sustainable, methods to produce biofuel and commodity chemicals. Cyanobacteria, like all phototrophs, can utilize the energy from sunlight to fix $\mathrm{CO}_{2}$ and produce a range of valuable carbon-based products via 'direct conversion' (1-3). The relatively high growth rate of these organisms, the use of water as the source of electrons and their genetic accessibility, has made them the preferred organisms for such applications.

Rhodopsins are light-sensitive seven-helix transmembrane proteins that bind a retinal molecule as their chromophore. This family has members with either a sensory or a chemiosmotic function in energy transduction. Bacteriorhodopsin from Halobacterium salinarum is the archetype of the chemiosmotic-energy-transducing rhodopsins (4). It pumps protons, driven by light, and hence is able to generate a proton motive force (pmf). Proteorhodopsins (PRs) form a subgroup of the rhodopsins from the Domain of the Bacteria, which utilize light energy to translocate protons over a membrane against an electrochemical proton gradient. The gene encoding the first discovered member of this group was detected in the genome sequence of an uncultured $\gamma$ proteobacterium from oceanic waters (5). Since then, PRs have turned out to be highly abundant in the oceans (6-9), and organisms containing them, including a cyanobacterium (10) can be found in many other habitats as well $(7,11-13)$. In vivo experiments have shown that pumping of protons by PR can lead to increases in growth rate under nutrient-limited conditions $(6,14,15)$, production of ATP (16), increased fixation of $\mathrm{CO}_{2}(6,17)$, and/or survival under starvation or stress conditions (16, 18-20). However, these enhancements generally require nutrient-limitation or stress conditions before they exceed experimental error.

PRs have also been heterologously expressed in non-photosynthetic hosts $(5,21-25)$. For example, the introduction of a PR in Shewanella oneidensis increased the pmf, which resulted in increases in electrical current generation, lactate uptake, and survival under starvation conditions $(23,24)$. In Escherichia coli, the pmf generated by a PR resulted in ATP synthesis (22), was able to drive the flagellar motor (21), and could be used to significantly increase the production of hydrogen by a co-introduced hydrogenase $(25,26)$. Very recently, functional expression of a proteorhodopsin in $E$. coli was shown to cause a minute increase the growth rate of this organism when growing fermentatively (27). This stimulation, however, may be strictly limited to anaerobic conditions: Photo-activation of Gloeobacter rhodopsin under aerobic conditions led to a decrease in growth rate of the organism because of increased oxidative stress (28).

Cyanobacteria and green algae have a significantly lower absorption in the green part of the visible spectrum (i.e. $450-550 \mathrm{~nm}$ ), a range that is well covered by the absorption spectrum of many PRs $(29,30)$. Introduction of PRs into such a system could lead to increased $\mathrm{CO}_{2}$ fixation, which could ultimately increase production rates of interesting compounds by cyanobacterial cell factories (31). Hence, it has previously been suggested that PRs could supply additional free energy to oxyphototrophic organisms $(29,30)$, even more so when it turns out to be possible to shift the window of absorption of this pigmented protein outside that of the PAR region (32). Several proposals have been made to increase the efficiency of oxygenic photosynthesis beyond its natural limits (e.g. (33)). In the most recent proposal (34) PSI is substituted by an (infrared-light dependent) proton pump; NADPH would then have to be derived via the combined action of PSII and NAD(P)H-dehydrogenase (34). Functional expression of PR may aid in the engineering towards obtaining such strains.

As a first step towards this we have expressed the gene sequence of the proteorhodopsin from Monterey Bay ((5); also known as GPR, but hereafter referred to as PR) in the cyanobacterium 
Synechocystis sp. PCC 6803 (hereafter Synechocystis). We first quantitated the amount of expressed apo-PR and its retinal chromophore, and characterized the sub-cellular distribution of the protein. Using the assay of growth (rate), we were then able to demonstrate an improvement in growth of the transgenic Synechocystis strain expressing PR, when compared with the control strain expressing a non-pumping proteorhodopsin, PROPS (35). To our knowledge, this is the first demonstration of a beneficial effect of PR on the growth of a cyanobacterium. By implication, we demonstrate for the first time that Synechocystis can synthesize all-trans-retinal in vivo.

\section{Materials \& Methods}

\section{Strains and growth conditions}

Strains of Escherichia coli were routinely grown at $37^{\circ} \mathrm{C}$, either in liquid LB medium while shaking at $200 \mathrm{rpm}$, or on solid LB plates containing $1.5 \%(\mathrm{w} / \mathrm{v})$ agar. Where appropriate, antibiotics were added to a final concentration of $100 \mu \mathrm{g} \cdot \mathrm{ml}^{-1}$ for ampicillin, and 25 to $50 \mu \mathrm{g} \cdot \mathrm{ml}^{-1}$ for kanamycin.

All Synechocystis sp. PCC 6803 strains used in this study were derived from a single wild-type strain (a glucose tolerant strain, obtained from D. Bhaya, Stanford University, Stanford, CA). Unless specified otherwise, Synechocystis was routinely grown at $30^{\circ} \mathrm{C}$ with continuous illumination by white light from fluorescent light sources at moderate intensities of approximately 30 to $50 \mu \mathrm{E} \cdot \mathrm{m}^{-2}$. $\mathrm{s}^{-1}\left(=\mu \mathrm{mol}\right.$ photons $\left.\cdot \mathrm{m}^{-2} \cdot \mathrm{s}^{-1}\right)$. Liquid cultures were grown in BG-11 medium (Sigma-Aldrich), supplemented with $50 \mathrm{mM}$ sodium bicarbonate, and incubated with shaking at $120 \mathrm{rpm}$. For growth on solid medium, BG-11 plates were made with 1.5\% (w/v) agar, $10 \mathrm{mM}$ TES-KOH (pH 8.0), $5 \mathrm{mM}$ glucose, and $0.3 \%(\mathrm{w} / \mathrm{v})$ sodium thiosulfate. Where appropriate, kanamycin was added at a final concentration of $20 \mu \mathrm{g} \cdot \mathrm{ml}^{-1}$ in plates, or $40 \mu \mathrm{g} \cdot \mathrm{ml}^{-1}$ in liquid medium.

Growth of Synechocystis sp. PCC 6803 was routinely monitored via the optical density at 730 $\mathrm{nm}\left(\mathrm{OD}_{730}\right)$ on a Biochrom WPA Lightwave II spectrophotometer. All light intensities mentioned were measured with a LI-COR LI-250 light meter and a LI-COR Quantum Sensor.

\section{Plasmid construction}

PCR reactions for cloning were performed with the proofreading Pwo DNA Polymerase (Roche Diagnostics). Primers are listed in Table I. All digestions made use of restriction enzymes from the FastDigest range (Thermo Scientific; formerly Fermentas). T4 DNA Ligase (Thermo Scientific) was used for ligations. Escherichia coli XL1-Blue (Agilent Technologies; formerly Stratagene) or MC1061 (36) were used as cloning hosts. Constructed plasmids were verified using specific PCRs with Taq DNA Polymerase (Thermo Scientific), followed by additional verification via sequencing.

The cloning plasmid pJBS1250 was constructed by introducing a terminator and several cloning sites into the broad-host-range conjugation vector pVZ321 (37). A fragment containing the bi-directional BBa_B0014 terminator was amplified from a template plasmid (31) using primers JBS300 and JBS301. The fragment was digested with Avrll and Pstl, and Dpnl was added to digest the template DNA. The plasmid backbone of pVZ321 was restricted with Xbal and Pstl, and treated with Fast Alkaline Phosphatase (FastAP; Thermo Scientific). This removed an approximately $1.7 \mathrm{~kb}$ region, including the chloramphenicol acetyl transferase (cat) gene. The digested plasmid and the PCR product were ligated to generate pJBS1250. Note that Xbal and Avrll form compatible overhangs of which the ligation product is not recognized by either of the two enzymes, which allows the same enzymes to be used again in subsequent cloning steps. 
The cloning plasmid was further extended with several promoter fragments, which all included a ribosome binding site (RBS), followed by an Avrll site (promoter-RBS-AvrlI). A fragment containing the trc promoter was amplified from template plasmid pSEQ-Trc (31) with primers JBS302 and JBS303. Similarly, fragments containing the $r n p B$ and the $p s b A 2$ promoters were amplified from template plasmids (38) with primers JBS339 and JBS340, and JBS341 and JBS342, respectively. All promoter-RBS-Avrll fragments were digested with EcoRI and Xbal. Plasmid pJBS1250 was digested with EcoRI and Spel, and treated with FastAp. The digested plasmid and fragments were subsequently ligated. Note that Xbal and Spel form compatible overhangs that do not result in the restoration of either restriction site after ligation. This procedure generated plasmids pJBS1253, with

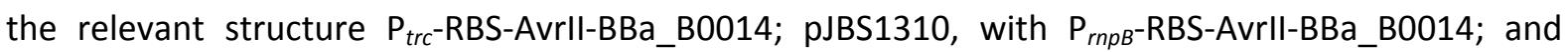
pJBS1312, with $\mathrm{P}_{\text {psbA2 }}$-RBS-Avrll-BBa_B0014.

The gene coding for proteorhodopsin (GenBank accession AF279106.2 (5)) with a C-terminal poly-histidine tag (PR-His) was amplified with primers JBS306 and JBS311 from plasmid pKJ900 (39), which was a kind gift of Dr. K. H. Jung from the University of Seoul, Korea, while the gene encoding proteorhodopsin optical proton sensor (PROPS, PR D97N (35)) was generated by introducing a G-A transversion in the PR gene using standard fusion PCR approach with primer pair JBS306/JBS319 and JBS320/JBS307. The resulting gene product was subsequently amplified with primers JBS306 and JBS307. The amplified fragment was digested with Xbal, and ligated into Avrll-digested pJBS1253, pJBS1310, or pJBS1312. This created plasmids pJBS1255, with the relevant structure $P_{t r c}-R B S-P R-H i s-$ BBa_B0014, pQC005, with $\mathrm{P}_{r n p B}-$ RBS-PR-His-BBa_B0014, and pQC006, with $\mathrm{P}_{p s b A 2}$-RBS-AvrllBBa_B0014.

\section{Conjugation}

Plasmids were transferred to Synechocystis sp. PCC 6803 via tri-parental mating, essentially as described before $(40)$. E. coli J53/RP4 $(41,42)$ was used as helper strain and E. coli XL1-Blue or MC1061 with the plasmid of interest was used as donor strain. For each strain, $10 \mathrm{ml}$ LB without antibiotics was inoculated with $250 \mu \mathrm{l}$ of an overnight culture. After $2.5 \mathrm{~h}$, the cultures were harvested by centrifugation. The pellets of the donor and the helper strains were subsequently resuspended in $1 \mathrm{ml}$ fresh LB each, mixed, and concentrated via centrifugation into $100 \mu \mathrm{l}$ fresh LB. The mixture was incubated at $30^{\circ} \mathrm{C}$ for $1 \mathrm{~h}$, after which $800 \mu \mathrm{l}$ of a young culture $\left(\mathrm{OD}_{730}<1.0\right)$ of Synechocystis sp. PCC 6803 was added. The three-strain mixture was centrifuged again, and the pellet was re-suspended in $30 \mu \mathrm{l}$ fresh BG-11 medium. The cells were spread on a Supor 200 sterile filter membrane with a pore-size of $0.2 \mu \mathrm{m}$ (Pall Corporation, Mexico) and placed on a BG-11 plate that had been supplemented with $5 \%(\mathrm{v} / \mathrm{v})$ Luria-Bertani medium. The plate was incubated overnight at $30^{\circ} \mathrm{C}$ under low-light conditions. After the incubation the membrane was removed from the plate, and cells were washed off with $300 \mu$ fresh BG-11 medium, which was then plated onto BG-11 plates with $20 \mu \mathrm{g} \cdot \mathrm{ml}^{-1}$ kanamycin. Single colonies were picked and plated again, after which independent single colonies were tested for the presence of the construct with a vector-specific and a construct-specific PCR.

\section{Western blots}

Synechocystis sp. PCC 6803 strains containing one of the plasmids described above were cultured under standard conditions with illumination at the intensity of approximately $50 \mu \mathrm{E} \cdot \mathrm{m}^{-2} \cdot \mathrm{s}^{-1}$. Cells were harvested by centrifugation and re-suspended into a $20 \mathrm{mM}$ sodium phosphate buffer at $\mathrm{pH} 8$ with $500 \mathrm{mM} \mathrm{NaCl}, 20 \mathrm{mM}$ imidazole, and $0.1 \%$ (w/v) n-dodecyl- $\beta$-D-maltopyranoside (DDM; 
GAMPRO Scientific). To this, $60 \%$ (w/v) 0.1-mm glass beads (Sigma-Aldrich) were added. The cells were subsequently disrupted in a Precellys 24 bead-beater (Bertin Technologies) by $20 \mathrm{~s}$ of beating at $6000 \mathrm{rpm}$, followed by $120 \mathrm{~s}$ on ice, which was repeated a total of ten times. An additional $15 \%$ $(\mathrm{w} / \mathrm{v})$ DDM was added to a final concentration of $1.5 \%(\mathrm{w} / \mathrm{v})$, and the cell lysates were incubated overnight at $4^{\circ} \mathrm{C}$ under mild agitation. Insoluble material and glass beads were removed by centrifugation at $10,000 \mathrm{~g}$ and $4^{\circ} \mathrm{C}$ for $15 \mathrm{~min}$. The protein concentration in the supernatant was determined with the Pierce BCA Protein Assay (Thermo Scientific) according to the manufacturer's protocol.

Proteins in the cell lysates were separated via SDS-PAGE in 'Any kD Mini-PROTEAN TGX Precast Gels' (Bio-Rad) using standard procedures. When bands from samples needed to be compared, equal amounts of protein ( $5 \mu \mathrm{g}$ unless specified otherwise) were loaded in each lane. The separated proteins were subsequently transferred to NitroPure nitrocellulose membranes (Osmonics). A pre-stained protein ladder (Thermo Scientific) was used to assess protein size and transfer efficiency. After transfer, the membranes were probed with monoclonal Anti-His ${ }_{6}$ antibodies (Roche) as primary antibody, and goat anti-mouse peroxidase (Thermo Scientific; formerly Pierce Biotechnology) as the secondary antibody. The bands were visualized using the SuperSignal West Femto Maximum Sensitivity Substrate Kit (Thermo Scientific) according to the manufacturer's protocol, and an Odyssey Fc imaging system (LI-COR).

For quantification, band intensities were determined using ImageJ v1.49a (W. S. Rasband, U.S. National Institutes of Health, http://imagej.nih.gov/ij/) by taking the total intensity of a band area corrected for the background intensity of an adjacent area of equal size. To determine the content of PR-His in a particular sample, its band intensity was compared with a standard curve formed by the band intensities of a series of known amounts of PR-His on the same gel. The standards were prepared from PR-His isolated from E. coli (43), which was quantified spectroscopically at its visible absorption maximum (525 $\mathrm{nm}$; see (44)). Relative PR content $(\% \mathrm{w} / \mathrm{w})$ in a sample was calculated from the PR-His protein content of the corresponding band over the total protein content loaded in the same lane. In order to present the PR-His expression level as the number of PR molecules per cell, the number of PR-His molecules in a particular band was calculated based on the PR-His protein content, the molar mass of PR-His $(28,359 \mathrm{~g} / \mathrm{mol}$ (calculated with ApE v2.0.45)) and Avogadro's number. Assuming that efficiency of cell disruption was $100 \%$, the total protein content loaded on a gel was converted into the number of cells. The former was determined with the Pierce BCA Protein Assay while the latter was estimated based on the $\mathrm{OD}_{730}$ of a sample, using the conversion factor that $1 \mathrm{ml}$ culture of wild type Synechocystis with an $\mathrm{OD}_{730}=1$ contains $10^{8}$ cells, as determined with a Casy 1 TTC cell counter (Schärfe System GmbH, Reutlingen, Germany) (45).

Images were minimally processed to increase printability using Adobe Photoshop CS5: they were cropped to show only the relevant areas, and brightness and contrast were adjusted equally over the entire image in some cases.

\section{Membrane fractionation}

Thylakoid and cytoplasmic membranes were isolated based on the procedure described in (46) from $20 \mathrm{I}$ batch cultures of Synechocystis (final $\mathrm{OD}_{730}=0.91$ ), with the modification that a two-phase system consisting of $6.7 \%$ dextran T-500 and $6.7 \%$ polyethylene glycol 3350 (both from SigmaAldrich) was used, instead of $5.8 \%$ and $5.8 \%$, respectively. The final membrane fractions were incubated overnight at $4^{\circ} \mathrm{C}$ under mild agitation in the presence of $1.5 \%(\mathrm{w} / \mathrm{v})$ DDM. His-tagged PR protein was subsequently detected as described under "Western blots" above. 
In this procedure the cytoplasmic membrane separates into two fractions: CM1, which may represent the attachment regions between the cytoplasmic and thylakoid membranes, and the main fraction CM2 (also referred to as PM1 and PM2, respectively) (46). Here, we only analyzed the main fraction of the cytoplasmic membrane (CM2).

The purity of the two separated membrane fractions was estimated using antibodies against two different markers: anti-PixJ1 $((47,48) ;$ a kind gift from Birgitta Norling (School of Biological Sciences, Nanyang Technological University, Singapore, Singapore), and anti-PsbC (CP43) (Agrisera, Vännäs, Sweden). PixJ1 has been reported to be a specific marker protein for the cytoplasmic membrane, while CP43 is a specific marker for the thylakoid membrane. The Western-blotting procedure used for these two marker proteins was similar to the detection procedure for His-tagged PR protein described above, except that the proteins were separated in SDS-PAGE gels with 14\% polyacrylamide plus $6 \mathrm{M}$ urea, transferred to PVDF membranes (Westran Clear Signal $0.45 \mu \mathrm{m}$, Whatman), and treated with goat anti-rabbit peroxidase (Thermo Scientific) as secondary antibody.

\section{Retinal identification and quantification}

Retinal was isolated, identified and quantified using a procedure adapted from (49). Cell pellets were re-suspended in $1 \mathrm{M}$ hydroxylamine at $\mathrm{pH} 8.0$ in $50 \%(\mathrm{v} / \mathrm{v})$ methanol, and disrupted via bead-beating as described above for the Western blot samples, prior to incubation at $30^{\circ} \mathrm{C}$ for $10 \mathrm{~min}$. During these steps (opsin-bound) retinal was converted with hydroxylamine into the more stable retinal oxime (50). The resulting reaction mixtures were subsequently extracted at least three times with petroleum ether $\left(40\right.$ to $60^{\circ} \mathrm{C}$ ). After pooling of the organic phases, the petroleum ether was evaporated under $\mathrm{N}_{2}$. The extracted material was then dissolved in n-heptane (HPLC grade), and separated on an HPLC system with an EC 150/4.6 NUCLEOSIL 100-5 C18 column (MACHEREY-NAGEL), and $\mathrm{n}$-heptane at $1 \mathrm{ml} \cdot \mathrm{min}^{-1}$ as mobile phase. Spectra of the eluting fractions were recorded using a UVD 340 U UV/VIS diode array (Thermo Scientific; formerly Dionex).

To aid peak identification, all-trans-retinal, all-trans-retinol, and retinoic acid (all from SigmaAldrich) were used as standard compounds. Retinal oxime was synthesized from all-trans-retinal by treatment with hydroxylamine, similar to the procedure used for the cell samples. For quantification, a dilution series of all-trans-retinal was treated exactly the same as the samples, including the beadbeating, incubation, and extraction steps, to include reaction and extraction efficiencies in the standard curve. Elution of retinal oxime was monitored at $357.7 \mathrm{~nm}$-the wavelength closest to its absorption maximum in the system used. The obtained peaks were integrated using (Chromeleon 6.80 SP1 Build 2238), and the peak areas were used to generate a standard curve. Standards were always processed together with the samples to account for any variation. The stock solution of alltrans-retinal was stored in n-heptane (HPLC grade) in darkness and was quantified spectroscopically before use at the visible absorption maximum of retinal (370 $\mathrm{nm}$ in $\mathrm{n}$-heptane (51)). To determine the retinal content in a sample, the peak area of retinal oxime in the sample was compared with a standard curve formed by the peak area of a series of known amounts of retinal (oxime). To present retinal production as the number of retinal molecules per cell, the number of retinal molecules was calculated based on retinal content, the mass of retinal $(284.4 \mathrm{~g} / \mathrm{mol})$ and Avogadro's number. The number of cells was estimated as described for the Western blotting procedure above.

\section{Isolation of proteorhodopsin from Synechocystis}

His-tagged PR was isolated from the same batch of cells as used for the membrane fractionation experiments (see above). Cells were harvested by centrifugation and disrupted by bead-beating as 
described for the Western blot samples above. After the removal of insoluble material by centrifugation, the lysate was diluted in buffer A $(20 \mathrm{mM}$ Tris- $\mathrm{HCl}, 500 \mathrm{mM} \mathrm{NaCl}, 20 \mathrm{mM}$ imidazole, $0.1 \% \mathrm{DDM}, \mathrm{pH} 8.0)$, and filtered using a syringe filter (0.22 $\mu \mathrm{m}$ pore size). His-tagged PR was subsequently purified using a HisTrap FF Crude column with $5 \mathrm{ml}$ column volume, and an ÄKTA FPLC system (all from GE Healthcare, Uppsala, Sweden). The protein was eluted with a gradient from buffer $A$ to buffer $B(20 \mathrm{mM}$ Tris- $\mathrm{HCl}, 500 \mathrm{mM} \mathrm{NaCl}, 500 \mathrm{mM}$ imidazole, $0.1 \%$ DDM, pH 8.0). Fractions containing the protein were collected, spectroscopically characterized, pooled, and immediately dialyzed against buffer $A$ to remove excess imidazole. After dialysis, the protein was concentrated using a concentrator with a molecular-weight cut-off of 10 kDa (Spin-X UF 6 Concentrator, Corning). The concentrator was also used to exchange the buffer for buffer $\mathrm{C}$ ( $20 \mathrm{mM}$ Tris- $\mathrm{HCl}, 100 \mathrm{mM} \mathrm{NaCl}, 0.1 \% \mathrm{DDM}, \mathrm{pH} 8.0$ ) by repeated cycles of washing and concentrating the protein.

The concentrated protein was further purified on a Superdex 200 HR 10/30 (GE Healthcare, Buckinghamshire, UK) gel filtration column with buffer $\mathrm{C}$ as mobile phase. Spectra of fractions were measured on an Agilent 8453 UV-Vis spectrophotometer (Agilent Technologies, Germany).

\section{Mass spectrometry}

Retinal was converted to retinal oxime and extracted using the same procedure as described above for the retinal identification and quantification. Fractions from the PR isolation (described above) and standards were treated in the same way. The resulting extract in n-heptane was dried under an argon flow, and re-dissolved in a mixture of $55 \%(\mathrm{v} / \mathrm{v})$ methanol, $30 \%(\mathrm{v} / \mathrm{v})$ acetonitrile and $0.1 \%$ $(\mathrm{v} / \mathrm{v})$ formic acid in water.

Mass spectrometric analysis was done with an AmaZon speed IonTrap (Bruker, Bremen, Germany) equipped with a nano-spray ESI source. Positive ion mode spectra between 50 and 600 $\mathrm{m} / \mathrm{z}$ were measured of samples and standards by direct sample infusion with a syringe pump system. The typical flow rate was $3 \mu \mathrm{l}$ per min. The $\mathrm{m} / \mathrm{z}$ values of compounds of interest were selected manually or by the automated $+\mathrm{MS}^{2}$ selection of the IonTrap in order to generate their MS/MS spectra. Collision-induced dissociation (CID) was used as the fragmentation method. Compass Data Analysis software (version 4.1; Bruker) was used to process and analyze the raw data. The pump system was extensively washed with $55 \%(\mathrm{v} / \mathrm{v})$ methanol, $30 \%(\mathrm{v} / \mathrm{v})$ acetonitrile and $0.1 \%(\mathrm{v} / \mathrm{v})$ formic acid in water after each run. A new analysis was not started until the background signal was restored to its initial value.

\section{Functional assays}

To determine the effect of PR expression on the growth of Synechocystis sp. PCC 6803, cells were grown in BG-11 medium with $50 \mathrm{mM}$ sodium bicarbonate grown at $30^{\circ} \mathrm{C}$ with shaking at $120 \mathrm{rpm}$ as usual (except for Figure 6B where PIPPS buffer was used rather than TES buffer), with the exception that cells were illuminated with light from a custom-made RGB (red, green, blue) light panel. This light panel was constructed using 40 LEDs of each color for 120 LEDs in total, and allowed tuning of the output light intensity of each color individually. The red, green, and blue LEDs emitted maximally at $635 \mathrm{~nm}, 527 \mathrm{~nm}$, and $459 \mathrm{~nm}$, respectively. The total light intensity used for these experiments was approximately $47 \mu \mathrm{E} \cdot \mathrm{m}^{-2} \cdot \mathrm{s}^{-1}$, made up out of $21 \mu \mathrm{E} \cdot \mathrm{m}^{-2} \cdot \mathrm{s}^{-1}$ red, $23 \mu \mathrm{E} \cdot \mathrm{m}^{-2} \cdot \mathrm{s}^{-1}$ green, and 3 $\mu \mathrm{E} \cdot \mathrm{m}^{-2} \cdot \mathrm{s}^{-1}$ blue light. To apply stress to the cells in the form of partial dissipation of the proton motive force sub-maximal concentrations of 3-(3,4-dichlorophenyl)-1,1-dimethylurea, DCMU), [3- 
chlorophenyl-hydrazono] malononitrile (CCCP) and sodium chloride were added to the BG-11 medium.

Three strains were compared in each experiment: cells containing the 'empty' plasmid pJBS1312, cells with the PR-His-encoding plasmid pQC006, and cells with the PROPS-encoding plasmid pQC011. To start an experiment, three 10-ml cultures were inoculated from a pre-culture for each strain in $50 \mathrm{ml}$ Erlenmeyer flasks in a shaking incubator at $30^{\circ} \mathrm{C}$. Growth was monitored via cell density by measuring the $\mathrm{OD}_{750}$ of a small volume $(150 \mu \mathrm{l})$ sample from each culture in a Multiskan FC Microplate Photometer (Thermo Scientific, Finland). For selected experiments, instead of $O D_{750}$, the number of cells per $\mathrm{ml}$ and average cell size was analyzed in triplicate with a Casy 1 Model TTC cell counter (Schärfe System GmbH, Reutlingen, Germany) with a $60 \mu \mathrm{m}$ capillary (52).

\section{Results}

\section{Expression of PR in Synechocystis}

To explore the possibility of functional expression of PR in Synechocystis, we constructed a series of expression plasmids based on the broad host-range conjugation vector pVZ321 (37). Each plasmid carried a promoter, a ribosome binding site, the structural gene of PR, with an extension encoding a C-terminal poly-histidine tag (PR-His), and a bi-directional terminator (see Materials \& Methods for further detail). We preferred a plasmid-based expression system over a genomic integration because this results in significantly (about 3-fold) higher expression levels for otherwise equal constructs (53).

To find the optimum expression level of PR, we compared three plasmids, each with a different promoter driving PR expression: the very strong trc promoter, the moderately strong $p s b A 2$ promoter, and the weak $r n p B$ promoter $(31,53,54)$. The protein-expression level of PR-His in strains carrying these three plasmids was determined using Western blots with an anti-His-tag antibody, and quantified by comparing the intensities of the PR-His bands with a standard series of known quantities of PR-His isolated from E. coli. The wild type strain was also included in this experiment, as a control to confirm that the labelling is specific for the His-tagged proteorhodopsin (data not shown).

Contrary to expectations, the highest level of PR-His expression was found in the strain in which its expression was controlled by the $p s b A 2$ promoter (data not shown). No expression could be demonstrated in the strain with $\mathrm{P}_{\text {trc }}$-driven expression, which was likely due to genetic instability of this construct, as after cultivation we were only able to re-isolate mutated plasmids from this strain. Although the cause of this genetic instability was not further investigated, it is plausible that very high PR expression levels poses a serious burden to the cells, which is not sufficiently compensated by energy conversion via $\mathrm{PR}$ and would provide a significant competitive advantage to spontaneous mutants impaired in PR expression. The construct with the weak $r n p B$ promoter did express PR-His, but levels were only detectable on Western blots after an additional concentration step of the cell-free extracts using a HisTrap spin column (data not shown). Therefore, we chose to utilize the $p s b A 2$ promoter for the remainder of this study.

To investigate the dependence of the PR-His expression level on the growth phase of Synechocystis growing in batch culture at a moderate light intensity $\left(\sim 50 \mu \mathrm{E} \cdot \mathrm{m}^{-2} \cdot \mathrm{s}^{-1}\right.$, see Materials \& Methods), we compared samples taken from different growth phases of such cultures. A representative result is shown in Legends to the figures:

Figure 1. We reproducibly found that the expression levels of PR-His rose slightly during the early growth phases, but declined again very late in the stationary phase. 


\section{Sub-cellular localization of PR in Synechocystis}

Cyanobacteria such as Synechocystis have two distinct inner membrane fractions: the cytoplasmic membrane (CM) and the thylakoid membrane (TM), be it that these two components may be partially connected $(48,55-57)$. Since our expression system did not include a specific membranetargeting signal, PR might localize in either one or in both of these membrane fractions. To investigate this, we fractionated the membranes of a PR-His expressing Synechocystis strain, using sucrose-density gradient centrifugation and a two-phase separation system (46). Of the two membrane fractions obtained equal amounts of protein (in $\mu \mathrm{g}$ ) were applied on SDS-PAGE, and analyzed using Western blots with three different antibodies: An anti-His-tag antibody to detect PRHis; an antibody against PsbC (CP43), a marker for the TM fraction (58); and an antibody against PixJ1, a marker specific for the CM fraction $(47,48)$.

The results obtained, summarized in

Figure 2, show that the level of PR-His relative to the total amount of protein in the respective fraction is comparable for both fractions (panel A). The marker proteins PsbC (panel B) and PixJ1 (panel $\mathrm{C}$ ) are almost exclusively isolated in the TM and the CM fraction, respectively, which shows that the fractions were separated successfully. Taken together, these results suggest that PR-His does not appear to localize to any particular membrane fraction selectively, but rather integrates into the $\mathrm{CM}$ and TM fraction randomly.

It should be noted that the membrane fractionation procedure we employed results in membrane fractions of high purity, but not in their quantitative isolation. Therefore, we have not made attempts to quantify the amount of PR-His in the two membrane fractions in vivo, beyond the conclusion that their level on a total protein basis is approximately the same in the two types of (purified) membrane (

Figure $2 \mathrm{~A})$.

\section{Retinal synthesis in Synechocystis}

The experiments described above established that the apo-PR protein can be expressed at significant levels in both major membrane fractions of Synechocystis. However, PR requires a retinal chromophore before it can become functional as a light-dependent proton pump. The gene product of sll1541 from Synechocystis has been shown to have apo-carotenoid-15,15'-oxygenase (SynACO) activity in vitro (59). The SynACO enzyme is able to convert $\beta$-apo-carotenals and related molecules of various chain-lengths into retinal in vitro (59). However, the most common precursor of retinal, $\beta$ carotene appears not to be accepted by SynACO as its substrate in vitro (59-61), which is probably due to the selectivity of the enzyme's substrate binding site $(61,62)$. It is not known whether or not the enzyme actually can produce retinal in vivo.

In order to test if Synechocystis has an endogenous pathway for synthesis of retinal, we adapted a retinal detection assay from (49) for analysis of samples from a batch culture. Briefly, cell extracts of Synechocystis were incubated with hydroxylamine, to allow formation of the stable retinal-derivative retinal oxime. This derivative was subsequently extracted using petroleum ether, and analyzed on a reverse-phase HPLC system, equipped with a $\mathrm{C}_{18}$ column and a diode-array detector (see Materials \& Methods for further details).

Despite repeated attempts, we were not able to detect any traces of retinal in wild type Synechocystis nor in those strains that carried an 'empty' control plasmid (i.e. without PR expression; 
pJBS1312; top trace in Figure 3A). Intriguingly, and in contrast to the results obtained with pJBS1312, we did detect a peak at the retention time of retinal oxime in extracts from cells that expressed PRHis (i.e. pQC006; middle trace in Figure 3A). In addition, the compound co-eluting with retinal oxime has a UV/Vis spectrum identical to retinal oxime (Figure 3B) and in contrast to visual pigments and bacteriorhodopsin (50) appears to be extracted predominantly in the syn form. These results strongly suggest that Synechocystis has the capacity to endogenously synthesize retinal, but that this compound is in most conditions rapidly metabolized. When apo-PR is present, however, the retinal can bind to this protein to form holo-PR. This presumably stabilizes the retinal against further metabolism. Thus, through the expression of apo-PR we have been able to demonstrate for the first time that Synechocystis can synthesize retinal in vivo.

Because the pathway of retinal synthesis in vivo has not been characterized yet, it is not clear whether retinal is present in similar amounts throughout all phases of growth. Therefore, we analyzed the content of retinal in the samples that had been used for quantification of the amount of apo-PR-His expressed (Legends to the figures:

Figure 1). Samples were extracted and analyzed as described in the legend to Figure 3. A known quantity of pure retinal was used to prepare a standard curve for each HPLC analysis experiment, which allowed an accurate quantification of the amount of retinal (oxime) that was isolated. The optical density at $730 \mathrm{~nm}\left(\mathrm{OD}_{730}\right)$ and the volume of the sample were used to estimate the number of cells from which this quantity of retinal was extracted, allowing the calculation of the approximate number of retinal molecules per cell (see Materials \& Methods for further details). It should be noted that this procedure uses several estimated conversion factors, and should be viewed as an approximation.

Similarly, the amount of PR-His protein in Western blot samples of the same cells (Legends to the figures:

Figure 1) was converted to an approximate number of PR molecules per cell. Like the estimation of the number of retinal molecules, also this procedure uses several estimated conversion factors (see Materials \& Methods for details), and should be viewed as an approximation. The number of apo-PR molecules and the number of retinal molecules in each of the five samples indicated by grey bars in Legends to the figures:

Figure 1 were estimated accordingly. We then calculated their ratio to vary between 0.9 and 2.1 (with an approximate error between biological replicates of 0.2 ) over the first three samples indicated in Figure 1. This ratio indicates that the amount of retinal available is in the right order of magnitude to allow the formation of detectable quantities of holo-PR. This conclusion is further supported by the isolation of pigmented PR from Synechocystis (see below). Surprisingly, at the end of the growth the amount of retinal in the cells increases steeply.

\section{Binding of retinal to apo-proteorhodopsin}

The appearance of a peak corresponding to retinal (oxime) in the HPLC traces of extracts from cells expressing PR (Figure 3 ) strongly suggests that retinal is associated with PR in vivo. To further confirm this, we made use of the His-tag on PR to isolate this protein using a HisTrap column. The resulting protein fraction had a bright pinkish color (inset in Figure 4A), as expected for holo-PR (5). 
The protein was further purified by molecular-sieve chromatography, and the absorption at $525 \mathrm{~nm}$ of all fractions was measured to determine the amount of holo-PR present in each sample. The resulting elution pattern showed that two main fractions were separated. In these two fractions $P R$ is presumably present in either of the two relevant oligomeric states, i.e. the hexameric and monomeric state, respectively, as is suggested by the absorption spectrum of the protein eluting in these two fractions (Figure 4B). The UV/Vis absorption spectrum of the material eluting in fraction $\mathrm{A}$ is the same as for holo-PR; the material eluting in fraction $B$ had a clearly red-shifted absorption maximum, consistent with the interpretation that this fraction contains monomeric PR (63) (see further Discussion).

To further confirm that the chromophore carried by the isolated and purified holo-protein indeed was retinal, we allowed the fractions isolated with the molecular-sieve chromatography (see Figure 4) to react with hydroxylamine, and extracted the chromophore in the same way as done for the whole-cell extraction described above (see Figure 3). The extracted chromophore was then analyzed with electrospray mass spectrometry, and compared with a standard. The MS/MS results obtained after selecting the mass of retinal oxime $(\mathrm{m} / \mathrm{z}$ 300.3) (Figure 5$)$ clearly show that several of its dominant fragmentation products are identical. It should be noted that the selection window for MS/MS will also include other compounds with a similar mass, resulting in co-fragmentation of other compounds that may differ between the fraction and standard. Thus, the results are not necessarily expected to be identical. However, in the corresponding fractions of strains that did not express PRHis, the $\mathrm{m} / \mathrm{z} 300.3$ signal with the fragmentation pattern as shown in Figure 5 was not detectable.

\section{Assay of functional activity of PR in vivo}

To test whether the expressed holo-PR would be active in vivo, and hence contribute to the organism's energy conversion, we first tried to demonstrate such physiological activity of PR by measuring light-driven proton translocation. However, whereas this approach worked successfully in starved E. coli cells expressing PR, it did not in Synechocystis. This was mainly because Synechocystis exhibits a number of competing light-dependent proton translocation and metabolic reactions which have an effect on the extracellular $\mathrm{pH}$, such as reactions involved in light-dependent electron transport and utilization of $\mathrm{CO}_{2} / \mathrm{HCO}_{3}{ }^{-}(64,65)$. It also turned out to be technically too challenging to demonstrate light-dependent proton translocation in isolated subcellular (thylakoid) membrane fractions of PR-expressing cells.

For that reason, we next attempted to demonstrate an effect of PR on the growth rate of Synechocystis. In order to reveal such a stimulatory effect, and because the presence of an expression plasmid may cause growth retardation, we compared the strains expressing PROPS (pQC011) and PR (pQC006) with the strain containing the 'empty' plasmid (pJBS1312). Furthermore, we used not only regular BG-11 medium, but also conditions that tend to abolish the pmf. For the latter, we chose several stresses that decrease the pmf: growth with only green light, in the presence of an inhibitor (3-(3,4-dichlorophenyl)-1,1-dimethylurea, DCMU) of photosynthesis; growth in the presence of a protonophorous uncoupler ([(3-chlorophenyl) hydrazono] malononitrile, CCCP), with and without glucose, and growth in a high salinity medium (66). These experiments were carried out with $47 \mu \mathrm{E} \cdot \mathrm{m}^{-2} \cdot \mathrm{s}^{-1} \mathrm{RGB}$ (red, green, blue) illumination, since green light is an indispensable element for PR-mediated proton translocation. Under standard conditions the control strain, i.e. Synechocystis containing the 'empty' plasmid pJBS1312, always had a higher growth rate than Synechocystis expressing PR (pQC006) or expressing the non-proton-pumping PROPS (i.e. the D97N mutant form of PR (35) from pQC011; see Figure 6A for an example). This is probably because 
expressing PR/PROPS caused an energy burden for Synechocystis, and/or PR/PROPS occupied a large fraction of the available space in the thylakoid membrane, which may have led to a impairment of photosynthetic electron flow. Consistent with this it was observed that the PR/PROPS expressing strains contain less chlorophyll and phycobilisomes (between 10 and $20 \%$ ) and these cells are slightly larger ( $\sim 10 \%$ increase of mean diameter) than cells expressing pJBS1312, during exponential growth in BG-11 medium. Significantly, these data also show that pQC006 allows Synechocystis to grow slightly faster than pQC011 (Figure 6A). To rule out that this faster growth would be due to alteration in light-scattering characteristics of the cells rather than to a true change in growth rate, the experiment was also analyzed via cell counting. Figure 6B shows that this analysis leads to the same conclusion.

In a significant number of experiments in which cells were grown with pmf-stress (see above), it was observed that the growth rate of the PR(pQc006)-containing strain was even slightly higher than the control strain (data not shown).

Our data demonstrates that PR can slightly enhance the rate of photoautotrophic growth of Synechocystis, relative to a strain that expresses similar amounts of non-functional proteorhodopsin. Under these conditions, PR presumably generates additional pmf, which may aid the cells in generating extra ATP for maintenance and/or anabolism.

\section{Discussion}

In this manuscript we report the heterologous (holo-protein) expression of a PR-based proton pump in the membranes of Synechocystis sp. PCC 6803. The heterologous protein incorporates approximately evenly (on a protein-content basis) in both types of membrane of the cells (i.e. CM and TM). It could also make a contribution to the cell's energy conversion in both membrane types. Furthermore, we demonstrated that this protein can make a measurable contribution to the conversion of solar energy into a useful form of metabolic energy for this organism (Figure 6). It is relevant to note that PR presumably also functions well at high-salt conditions (67).

Expression of this proteorhodopsin was so far only directly detectable when using the psbA2 promoter, which is of intermediate strength. With weaker promoters, detection of the protein with Western blots required specific pre-concentration steps, whereas the stronger trc promoter elicited genetic instability of the heterologous gene. The expression level of the apo-protein peaks at the late stages of (linear) growth in the batch cultures studied here, and then declines during stationary phase. A similar growth-phase dependence of PR expression was observed in the naturally PRcontaining Dokdonia sp. MED134 (6). The drop in PR content during the stationary phase may be in part due to the promoter we selected, because it is known that psbA2 expression and transcript stability are light-intensity dependent (68-73), and so is heterologous expression driven by this promoter $(74,75)$. It is worth noting that several of the recently discovered proteorhodopsins in 'chemotrophic', often psychrophilic, bacteria only contribute detectably to growth rate or cell yield when the cells are stressed and/or in stationary phase $(6,14-16,18-20)$.

Beyond the apo-protein, Synechocystis sp. PCC 6803 also turned out to be able to synthesize its natural chromophore, retinal, which spontaneously forms a Schiff-base linkage with the apo-PR to form the holo-protein (Figure 3-5). This fraction of protein-bound retinal then becomes protected against catabolism, which is the regular fate of both endogenously and exogenously-added retinal in this organism: micromolar concentrations of retinal added to a batch culture of Synechocystis are 
catabolized with a half time of between 1 and $2 \mathrm{~h}$ (data not shown). Synthesis and degradation of retinal have been studied before in Synechocystis, but only via in vitro enzyme assays $(59-61,76,77)$.

Upon isolation of the heterologously expressed PR from Synechocystis, two distinct fractions of the protein were obtained with different (spectral) characteristics (Figure 4). We interpret these differences as being a consequence of the oligomeric state of the protein, consistent with the results presented by Hussain et al. (63). These authors showed convincingly that the dominant form of organization of (heterologously expressed) PR molecules after solubilization in DDM is the quaternary structure of a hexameric ring, whereas a small fraction is isolated as monomeric PR molecules. They also reported a higher pKa for the monomeric state, which resulted in a red-shifted absorption spectrum as compared to the hexameric state ( $\lambda_{\max } 518 \mathrm{~nm}$, alkaline, vs. $535 \mathrm{~nm}$, acidic), which we also observed here. We therefore tentatively conclude that PR is predominantly present in a hexameric organization, both in the thylakoid and in the cytoplasmic membranes of Synechocystis. The small amount of monomeric PR may actually be caused by detergent solubilization $(63,78)$.

To show that PR is able to make a contribution to light-energy conversion in Synechocystis, we also made use of starvation/stressful conditions, just like it was initially done in E. coli (21) and in some of the organisms in which it is endogenously present $(6,14-16,18-20)$. However, no fully consistent data have been obtained under these conditions so far. This is because the technical reproducibility of these experiments was less than with growth in BG-11 medium. The small contribution of PR to the energy metabolism in Synechocystis observed here will in part be due to its relatively slow turnover (i.e. 'pumping') rate, which may be close to only 10 protons per second (7981). Taking the latter value as a starting point one can calculate that in Synechocystis, growing with a doubling time of about $8 \mathrm{~h}$ and carrying out predominantly linear electron flow, the retinal-based pump, when expressed at levels of up to $10^{5}$ molecules per cell, contributes to proton motive force generation (and hence ATP production) less than $1 \%$ (this is assuming that one $\mathrm{CO}_{2}$ fixed requires the pumping of 10 protons). Furthermore, we consistently observed that expression of the heterologous membrane protein PR-His decreased the growth rate of Synechocystis sp. PCC 6803. Therefore, use of the proper control strain (i.e. the strain carrying PROPS (pQC011)) is crucial in these experiments. The calculation of the $1 \%$ stimulation is based on many simplifying assumptions, like neglect of the multiple (at least 6; see (82)) forms of cyclic electron flow. This positive effect, however, may be offset by increased formation of reactive oxygen intermediates, as recently shown in $E$. coli (28), because cyanobacteria are very sensitive to $\mathrm{H}_{2} \mathrm{O}_{2}$ (83).

To further increase the potential of retinal phototrophy in Synechocystis various possibilities can be proposed. The first is to use a proteorhodopsin variant that pumps faster. Since the start of this project several routes towards improvement along this line have revealed themselves, like the use of proteorhodopsin variants that bind a carotenoid like echinenone (84), not only because of their pumping rate (32), but also because their antenna carotenoid increases their effective absorption cross section 3.5 fold (85). A second possibility may be to use the trimeric bacteriorhodopsin, which presumably will not form an extended hexagonal lattice, but will show a higher pumping rate. Bacteriorhodopsin, the archetype of the retinal-based proton pumps and retinal-based photosynthesis (4), pumps with a rate of about 100 protons per second (86). The recent description of a sodium-ion translocating bacterial rhodopsin (87) offers even more exciting opportunities to explore in relation to salt stress and phototrophy. It should be kept in mind that there is one example of an endogenous proteorhodopsin in a cyanobacterium: Gloeobacter violaceus contains a retinal-based proton pump next to its regular machinery for oxygenic 
photosynthesis (10). However, the physiological role of this rhodopsin has not been resolved (yet; see (88)).

The contribution of retinal-based proton pumping to the energy budget of Synechocystis would become much more significant once it would be possible to exploit the photons that are not utilized by wild-type cyanobacteria. For this, the absorbance maximum of the proteorhodopsin would have to be shifted to beyond $700 \mathrm{~nm}$. Although progress is made in this direction (32) there is a significant way still to go. In a seminal paper, written at the peak of the recent revival of interest in sustainability research (33), a comparison of 'artificial' and natural photosynthesis was made, together with a review of options to increase the efficiency of light energy conversion through rational engineering in both approaches. Although the possibility to exploit retinal photosynthesis is not mentioned, the option to use the near-infra-red part of the solar spectrum is recommended, in particular through exploiting cyanobacteria that synthesize chlorophyll $d$ or $f$ and/or further engineering pigment synthesis. In that paper the possibility to alter the free-energy gap to excite the two photosystems is also proposed as a way to exploit a larger part of the solar spectrum. However, those approaches have the disadvantage that they strongly reduce the amount of free energy available to drive electron transfer between the two photosystems. Such a near-infra-red light driven retinal-based proton pump will have sufficient free energy available to drive proton translocation at physiological levels of the proton motive force. Bacteriorhodopsin pumps only a single proton per photon $(89,90)$, whereas in purple bacteria like Rhodopseudomonas viridis photons with a wavelength close to $1,000 \mathrm{~nm}$ provide the organism with enough free energy to translocate two protons over their cytoplasmic membrane (see also (30)).

In a recent update of the discussion paper on the efficiency of oxygenic photosynthesis (34) it is proposed to cut electron transfer through the Z-scheme into two halves, which then should lead to: (i) linear electron flow via PS-II plus NDH-1 for water-driven reduction of NADPH, and (ii) replacement of PS-I by an infra-red absorbing system for proton pumping, like e.g. a cyclic electron transfer chain as it functions in purple-non-sulfur bacteria. This would then allow exploitation of all solar radiation for oxygenic photosynthesis. Functional expression of a proteorhodopsin in a PS-I deletion strain may be a very significant step in such an engineering approach.

Strong emphasis on maximizing efficiency is necessary if natural photosynthesis is to be used for large-scale fuel production, e.g. via (cyanobacterial) cell factories. In this approach, however, there are many more aspects that can be further optimized, like e.g. expression of an improved RuBisCO (91), antenna truncation $(92,93)$, and a retinal-based proton pump to modulate the relative rate of ATP and NADPH synthesis and stimulate product formation (94).

\section{Authors' Contributions}

JBvdS, QC and KJH designed experiments; QC, JBvdS and HLD performed experiments; JBvdS, QC and $\mathrm{KJH}$ wrote the paper; and SG and WJdG contributed to the writing of the paper and the overall experimental design.

\section{Conflict of Interest}

The authors declare that they have no conflict of interest. $\mathrm{KJH}$ is scientific advisor to the start-up company Photanol BV. This does not create a conflict of interest nor does it alter the authors' adherence to accepted policies on sharing data and materials. 


\section{Acknowledgements}

This project was carried out within the research program of BioSolar Cells (BSC core project grant C2.9 to WJdG and KJH), co-financed by the Dutch Ministry of Economic Affairs. The authors would like to acknowledge Jos C. Arents and Louis Hartog for their help with experiments. 


\section{References}

1. Angermayr, S. A., K. J. Hellingwerf, P. Lindblad, and M. J. Teixeira de Mattos. 2009. Energy biotechnology with cyanobacteria. Curr. Opin. Biotechnol. 20:257-263. doi:

10.1016/j.copbio.2009.05.011.

2. Ducat, D. C., J. C. Way, and P. A. Silver. 2011. Engineering cyanobacteria to generate high-value products. Trends Biotechnol. 29:95-103. doi: 10.1016/j.tibtech.2010.12.003;

10.1016/j.tibtech.2010.12.003.

3. Machado, I. M., and S. Atsumi. 2012. Cyanobacterial biofuel production. J. Biotechnol. 162:50-56. doi: 10.1016/j.jbiotec.2012.03.005.

4. Lanyi, J. K. 1978. Light energy conversion in Halobacterium halobium. Microbiol. Rev. 42:682-706.

5. Beja, O., L. Aravind, E. V. Koonin, M. T. Suzuki, A. Hadd, L. P. Nguyen, S. B. Jovanovich, C. M. Gates, R. A. Feldman, J. L. Spudich, E. N. Spudich, and E. F. DeLong. 2000. Bacterial rhodopsin: evidence for a new type of phototrophy in the sea. Science. 289:1902-1906.

6. Palovaara, J., N. Akram, F. Baltar, C. Bunse, J. Forsberg, C. Pedros-Alio, J. M. Gonzalez, and J. Pinhassi. 2014. Stimulation of growth by proteorhodopsin phototrophy involves regulation of central metabolic pathways in marine planktonic bacteria. Proc. Natl. Acad. Sci. U. S. A. 111:E3650-8. doi: 10.1073/pnas.1402617111 [doi].

7. Rusch, D. B., A. L. Halpern, G. Sutton, K. B. Heidelberg, S. Williamson, S. Yooseph, D. Wu, J. A. Eisen, J. M. Hoffman, K. Remington, K. Beeson, B. Tran, H. Smith, H. Baden-Tillson, C. Stewart, J. Thorpe, J. Freeman, C. Andrews-Pfannkoch, J. E. Venter, K. Li, S. Kravitz, J. F. Heidelberg, T. Utterback, Y. H. Rogers, L. I. Falcon, V. Souza, G. Bonilla-Rosso, L. E. Eguiarte, D. M. Karl, S. Sathyendranath, T. Platt, E. Bermingham, V. Gallardo, G. Tamayo-Castillo, M. R. Ferrari, R. L. Strausberg, K. Nealson, R. Friedman, M. Frazier, and J. C. Venter. 2007. The Sorcerer II Global Ocean Sampling expedition: northwest Atlantic through eastern tropical Pacific. PLoS Biol. 5:e77. doi: 10.1371/journal.pbio.0050077.

8. Finkel, O. M., O. Beja, and S. Belkin. 2013. Global abundance of microbial rhodopsins. Isme J. 7:448-451. doi: 10.1038/ismej.2012.112 [doi].

9. Campbell, B. J., L. A. Waidner, M. T. Cottrell, and D. L. Kirchman. 2008. Abundant proteorhodopsin genes in the North Atlantic Ocean. Environ. Microbiol. 10:99-109. doi: 10.1111/j.1462-2920.2007.01436.x.

10. Miranda, M. R., A. R. Choi, L. Shi, A. G. Bezerra Jr, K. H. Jung, and L. S. Brown. 2009. The photocycle and proton translocation pathway in a cyanobacterial ion-pumping rhodopsin. Biophys. J. 96:1471-1481. doi: 10.1016/j.bpj.2008.11.026.

11. Atamna-Ismaeel, N., G. Sabehi, I. Sharon, K. P. Witzel, M. Labrenz, K. Jurgens, T. Barkay, M. Stomp, J. Huisman, and O. Beja. 2008. Widespread distribution of proteorhodopsins in freshwater and brackish ecosystems. Isme J. 2:656-662. doi: 10.1038/ismej.2008.27.

12. Atamna-Ismaeel, N., O. M. Finkel, F. Glaser, I. Sharon, R. Schneider, A. F. Post, J. L. Spudich, C. von Mering, J. A. Vorholt, D. Iluz, O. Beja, and S. Belkin. 2012. Microbial rhodopsins on leaf surfaces 
of terrestrial plants. Environ. Microbiol. 14:140-146. doi: 10.1111/j.1462-2920.2011.02554.x; 10.1111/j.1462-2920.2011.02554.x.

13. Koh, E. Y., N. Atamna-Ismaeel, A. Martin, R. O. Cowie, O. Beja, S. K. Davy, E. W. Maas, and K. G. Ryan. 2010. Proteorhodopsin-bearing bacteria in Antarctic sea ice. Appl. Environ. Microbiol. 76:5918-5925. doi: 10.1128/AEM.00562-10 [doi].

14. Gomez-Consarnau, L., J. M. Gonzalez, M. Coll-Llado, P. Gourdon, T. Pascher, R. Neutze, C. Pedros-Alio, and J. Pinhassi. 2007. Light stimulates growth of proteorhodopsin-containing marine Flavobacteria. Nature. 445:210-213. doi: 10.1038/nature05381.

15. Kimura, H., C. R. Young, A. Martinez, and E. F. Delong. 2011. Light-induced transcriptional responses associated with proteorhodopsin-enhanced growth in a marine flavobacterium. Isme J. 5:1641-1651. doi: 10.1038/ismej.2011.36; 10.1038/ismej.2011.36.

16. Steindler, L., M. S. Schwalbach, D. P. Smith, F. Chan, and S. J. Giovannoni. 2011. Energy starved Candidatus Pelagibacter ubique substitutes light-mediated ATP production for endogenous carbon respiration. PLoS One. 6:e19725. doi: 10.1371/journal.pone.0019725.

17. Gonzalez, J. M., B. Fernandez-Gomez, A. Fernandez-Guerra, L. Gomez-Consarnau, O. Sanchez, M. Coll-Llado, J. Del Campo, L. Escudero, R. Rodriguez-Martinez, L. Alonso-Saez, M. Latasa, I. Paulsen, O. Nedashkovskaya, I. Lekunberri, J. Pinhassi, and C. Pedros-Alio. 2008. Genome analysis of the proteorhodopsin-containing marine bacterium Polaribacter sp. MED152 (Flavobacteria). Proc. Natl. Acad. Sci. U. S. A. 105:8724-8729. doi: 10.1073/pnas.0712027105.

18. Gomez-Consarnau, L., N. Akram, K. Lindell, A. Pedersen, R. Neutze, D. L. Milton, J. M. Gonzalez, and J. Pinhassi. 2010. Proteorhodopsin phototrophy promotes survival of marine bacteria during starvation. PLoS Biol. 8:e1000358. doi: 10.1371/journal.pbio.1000358.

19. Akram, N., J. Palovaara, J. Forsberg, M. V. Lindh, D. L. Milton, H. Luo, J. M. Gonzalez, and J. Pinhassi. 2013. Regulation of proteorhodopsin gene expression by nutrient limitation in the marine bacterium Vibrio sp. AND4. Environ. Microbiol. 15:1400-1415. doi: 10.1111/1462-2920.12085; 10.1111/1462-2920.12085.

20. Wang, Z., T. J. O'Shaughnessy, C. M. Soto, A. M. Rahbar, K. L. Robertson, N. Lebedev, and G. J. Vora. 2012. Function and regulation of Vibrio campbellii proteorhodopsin: acquired phototrophy in a classical organoheterotroph. PLoS One. 7:e38749. doi: 10.1371/journal.pone.0038749 [doi].

21. Walter, J. M., D. Greenfield, C. Bustamante, and J. Liphardt. 2007. Light-powering Escherichia coli with proteorhodopsin. Proc. Natl. Acad. Sci. U. S. A. 104:2408-2412. doi:

10.1073/pnas.0611035104.

22. Martinez, A., A. S. Bradley, J. R. Waldbauer, R. E. Summons, and E. F. DeLong. 2007. Proteorhodopsin photosystem gene expression enables photophosphorylation in a heterologous host. Proc. Natl. Acad. Sci. U. S. A. 104:5590-5595. doi: 10.1073/pnas.0611470104.

23. Johnson, E. T., D. B. Baron, B. Naranjo, D. R. Bond, C. Schmidt-Dannert, and J. A. Gralnick. 2010. Enhancement of survival and electricity production in an engineered bacterium by light-driven proton pumping. Appl. Environ. Microbiol. 76:4123-4129. doi: 10.1128/AEM.02425-09. 
24. Hunt, K. A., J. M. Flynn, B. Naranjo, I. D. Shikhare, and J. A. Gralnick. 2010. Substrate-level phosphorylation is the primary source of energy conservation during anaerobic respiration of Shewanella oneidensis strain MR-1. J. Bacteriol. 192:3345-3351. doi: 10.1128/JB.00090-10.

25. Kim, J. Y., B. H. Jo, Y. Jo, and H. J. Cha. 2012. Improved production of biohydrogen in lightpowered Escherichia coli by co-expression of proteorhodopsin and heterologous hydrogenase. Microb. Cell. Fact. 11:2. doi: 10.1186/1475-2859-11-2.

26. Kuniyoshi, T. M., A. Balan, A. C. Schenberg, D. Severino, and P. C. Hallenbeck. 2015. Heterologous expression of proteorhodopsin enhances $\mathrm{H}$ production in Escherichia coli when endogenous Hyd-4 is overexpressed. J. Biotechnol. 206:52-57. doi: S0168-1656(15)00174-1 [pii].

27. Wang, Y., Y. Li, T. Xu, Z. Shi, and Q. Wu. 2015. Experimental evidence for growth advantage and metabolic shift stimulated by photophosphorylation of proteorhodopsin expressed in Escherichia coli at anaerobic condition. Biotechnol. Bioeng. 112:947-956. doi: 10.1002/bit.25504 [doi].

28. Na, Y. A., J. Y. Lee, W. J. Bang, H. J. Lee, S. I. Choi, S. K. Kwon, K. H. Jung, J. F. Kim, and P. Kim. 2015. Growth retardation of Escherichia coli by artificial increase of intracellular ATP. J. Ind. Microbiol. Biotechnol. 42:915-924. doi: 10.1007/s10295-015-1609-6 [doi].

29. Walter, J. M., D. Greenfield, and J. Liphardt. 2010. Potential of light-harvesting proton pumps for bioenergy applications. Curr. Opin. Biotechnol. 21:265-270. doi: 10.1016/j.copbio.2010.03.007.

30. Claassens, N. J., M. Volpers, V. A. dos Santos, J. van der Oost, and W. M. de Vos. 2013. Potential of proton-pumping rhodopsins: engineering photosystems into microorganisms. Trends Biotechnol. 31:633-642. doi: 10.1016/j.tibtech.2013.08.006 [doi].

31. Angermayr, S. A., M. Paszota, and K. J. Hellingwerf. 2012. Engineering a cyanobacterial cell factory for the production of lactic acid. Appl. Environ. Microbiol. 78:7098-7106. doi: 10.1128/AEM.01587-12.

32. Ganapathy, S., O. Becheau, H. Venselaar, S. Frolich, J. B. van der Steen, Q. Chen, S. Radwan, J. Lugtenburg, K. J. Hellingwerf, H. J. de Groot, and W. J. de Grip. 2015. Modulation of spectral properties and pump activity of proteorhodopsins by retinal analogues. Biochem. J. 467:333-343. doi: 10.1042/BJ20141210 [doi].

33. Blankenship, R. E., D. M. Tiede, J. Barber, G. W. Brudvig, G. Fleming, M. Ghirardi, M. R. Gunner, W. Junge, D. M. Kramer, A. Melis, T. A. Moore, C. C. Moser, D. G. Nocera, A. J. Nozik, D. R. Ort, W. W. Parson, R. C. Prince, and R. T. Sayre. 2011. Comparing photosynthetic and photovoltaic efficiencies and recognizing the potential for improvement. Science. 332:805-809. doi: 10.1126/science.1200165 [doi].

34. Ort, D. R., S. S. Merchant, J. Alric, A. Barkan, R. E. Blankenship, R. Bock, R. Croce, M. R. Hanson, J. M. Hibberd, S. P. Long, T. A. Moore, J. Moroney, K. K. Niyogi, M. A. Parry, P. P. Peralta-Yahya, R. C. Prince, K. E. Redding, M. H. Spalding, K. J. van Wijk, W. F. Vermaas, S. von Caemmerer, A. P. Weber, T. O. Yeates, J. S. Yuan, and X. G. Zhu. 2015. Redesigning photosynthesis to sustainably meet global food and bioenergy demand. Proc. Natl. Acad. Sci. U. S. A. 112:8529-8536. doi: 10.1073/pnas.1424031112 [doi]. 
35. Kralj, J. M., D. R. Hochbaum, A. D. Douglass, and A. E. Cohen. 2011. Electrical spiking in Escherichia coli probed with a fluorescent voltage-indicating protein. Science. 333:345-348. doi: 10.1126/science.1204763.

36. Casadaban, M. J., and S. N. Cohen. 1980. Analysis of gene control signals by DNA fusion and cloning in Escherichia coli. J. Mol. Biol. 138:179-207.

37. Zinchenko, V. V., I. V. Piven, V. A. Melnik, and S. V. Shestakov. 1999. Vectors for the Complementation Analysis of Cyanobacterial Mutants. Russ. J. Genet. 35:228-232.

38. Angermayr, S. A., and K. J. Hellingwerf. 2013. On the use of metabolic control analysis in the optimization of cyanobacterial biosolar cell factories. J Phys Chem B. 117:11169-11175. doi: 10.1021/jp4013152; 10.1021/jp4013152.

39. Kim, S. Y., S. A. Waschuk, L. S. Brown, and K. H. Jung. 2008. Screening and characterization of proteorhodopsin color-tuning mutations in Escherichia coli with endogenous retinal synthesis. Biochim. Biophys. Acta. 1777:504-513. doi: 10.1016/j.bbabio.2008.03.010.

40. Savakis, P. E., S. A. Angermayr, and K. J. Hellingwerf. 2013. Synthesis of 2,3-butanediol by Synechocystis sp. PCC6803 via heterologous expression of a catabolic pathway from lactic acid- and enterobacteria. Metab. Eng. 20:121-130. doi: 10.1016/j.ymben.2013.09.008.

41. Bachmann, B. J. 1972. Pedigrees of some mutant strains of Escherichia coli K-12. Bacteriol. Rev. 36:525-557.

42. Jacob, A. E., and N. J. Grinter. 1975. Plasmid RP4 as a vector replicor in genetic engineering. Nature. 255:504-506.

43. Rupenyan, A., I. H. van Stokkum, J. C. Arents, R. van Grondelle, K. J. Hellingwerf, and M. L. Groot. 2009. Reaction pathways of photoexcited retinal in proteorhodopsin studied by pump-dumpprobe spectroscopy. J Phys Chem B. 113:16251-16256. doi: 10.1021/jp9065289 [doi].

44. Rupenyan, A., I. H. van Stokkum, J. C. Arents, R. van Grondelle, K. Hellingwerf, and M. L. Groot. 2008. Characterization of the primary photochemistry of proteorhodopsin with femtosecond spectroscopy. Biophys. J. 94:4020-4030. doi: 10.1529/biophysj.107.121376 [doi].

45. Schuurmans, R. M., J. M. Schuurmans, M. Bekker, J. C. Kromkamp, H. C. Matthijs, and K. J. Hellingwerf. 2014. The redox potential of the plastoquinone pool of the cyanobacterium Synechocystis species strain PCC 6803 is under strict homeostatic control. Plant Physiol. 165:463475. doi: 10.1104/pp.114.237313 [doi].

46. Huang, F., I. Parmryd, F. Nilsson, A. L. Persson, H. B. Pakrasi, B. Andersson, and B. Norling. 2002. Proteomics of Synechocystis sp. strain PCC 6803: identification of plasma membrane proteins. Mol. Cell. Proteomics. 1:956-966.

47. Selao, T. T., L. Zhang, C. Arioz, A. Wieslander, and B. Norling. 2014. Subcellular localization of monoglucosyldiacylglycerol synthase in Synechocystis sp. PCC6803 and its unique regulation by lipid environment. PLoS One. 9:e88153. doi: 10.1371/journal.pone.0088153 [doi].

48. Pisareva, T., J. Kwon, J. Oh, S. Kim, C. Ge, A. Wieslander, J. S. Choi, and B. Norling. 2011. Model for membrane organization and protein sorting in the cyanobacterium Synechocystis sp. PCC 6803 
inferred from proteomics and multivariate sequence analyses. J. Proteome Res. 10:3617-3631. doi: $10.1021 / p r 200268$ r [doi].

49. von Lintig, J., and K. Vogt. 2000. Filling the gap in vitamin A research. Molecular identification of an enzyme cleaving beta-carotene to retinal. J. Biol. Chem. 275:11915-11920.

50. Groenendijk, G. W., W. J. De Grip, and F. J. Daemen. 1980. Quantitative determination of retinals with complete retention of their geometric configuration. Biochim. Biophys. Acta. 617:430438.

51. Hubbard, R. 1966. The stereoisomerization of 11-cis-retinal. J. Biol. Chem. 241:1814-1818.

52. Sandrini, G., D. Jakupovic, H. C. Matthijs, and J. Huisman. 2015. Strains of the Harmful Cyanobacterium Microcystis aeruginosa Differ in Gene Expression and Activity of Inorganic Carbon Uptake Systems at Elevated CO2 Levels. Appl. Environ. Microbiol. 81:7730-7739. doi: 10.1128/AEM.02295-15 [doi].

53. Berla, B. M., R. Saha, C. M. Immethun, C. D. Maranas, T. S. Moon, and H. B. Pakrasi. 2013. Synthetic biology of cyanobacteria: unique challenges and opportunities. Front. Microbiol. 4:246. doi: 10.3389/fmicb.2013.00246 [doi].

54. Huang, H. H., D. Camsund, P. Lindblad, and T. Heidorn. 2010. Design and characterization of molecular tools for a Synthetic Biology approach towards developing cyanobacterial biotechnology. Nucleic Acids Res. 38:2577-2593. doi: 10.1093/nar/gkq164.

55. Liberton, M., R. Howard Berg, J. Heuser, R. Roth, and H. B. Pakrasi. 2006. Ultrastructure of the membrane systems in the unicellular cyanobacterium Synechocystis sp. strain PCC 6803. Protoplasma. 227:129-138. doi: 10.1007/s00709-006-0145-7 [doi].

56. Schneider, D., E. Fuhrmann, I. Scholz, W. R. Hess, and P. L. Graumann. 2007. Fluorescence staining of live cyanobacterial cells suggest non-stringent chromosome segregation and absence of a connection between cytoplasmic and thylakoid membranes. BMC Cell Biol. 8:39. doi: 1471-2121-839 [pii].

57. van de Meene, A. M., M. F. Hohmann-Marriott, W. F. Vermaas, and R. W. Roberson. 2006. The three-dimensional structure of the cyanobacterium Synechocystis sp. PCC 6803. Arch. Microbiol. 184:259-270. doi: 10.1007/s00203-005-0027-y [doi].

58. Norling, B., E. Zak, B. Andersson, and H. Pakrasi. 1998. 2D-isolation of pure plasma and thylakoid membranes from the cyanobacterium Synechocystis sp. PCC 6803. FEBS Lett. 436:189-192.

59. Ruch, S., P. Beyer, H. Ernst, and S. Al-Babili. 2005. Retinal biosynthesis in Eubacteria: in vitro characterization of a novel carotenoid oxygenase from Synechocystis sp. PCC 6803. Mol. Microbiol. 55:1015-1024. doi: 10.1111/j.1365-2958.2004.04460.x.

60. Marasco, E. K., K. Vay, and C. Schmidt-Dannert. 2006. Identification of carotenoid cleavage dioxygenases from Nostoc sp. PCC 7120 with different cleavage activities. J. Biol. Chem. 281:3158331593. doi: 10.1074/jbc.M606299200. 
61. Sui, X., P. D. Kiser, T. Che, P. R. Carey, M. Golczak, W. Shi, J. Von Lintig, and K. Palczewski. 2014. Analysis of carotenoid isomerase activity in a prototypical carotenoid cleavage enzyme, apocarotenoid oxygenase (ACO). J. Biol. Chem. . doi: 10.1074/jbc.M114.552836.

62. Kloer, D. P., and G. E. Schulz. 2006. Structural and biological aspects of carotenoid cleavage. Cell Mol. Life Sci. 63:2291-2303. doi: 10.1007/s00018-006-6176-6.

63. Hussain, S., M. Kinnebrew, N. S. Schonenbach, E. Aye, and S. Han. 2015. Functional consequences of the oligomeric assembly of Proteorhodopsin. J. Mol. Biol. . doi: S00222836(15)00009-1 [pii].

64. Katoh, A., M. Sonoda, H. Katoh, and T. Ogawa. 1996. Absence of light-induced proton extrusion in a cotA-less mutant of Synechocystis sp. strain PCC6803. J. Bacteriol. 178:5452-5455.

65. Sonoda, M., H. Katoh, W. Vermaas, G. Schmetterer, and T. Ogawa. 1998. Photosynthetic electron transport involved in PxcA-dependent proton extrusion in Synechocystis sp. Strain PCC6803: effect of pxcA inactivation on CO2, HCO3-, and NO3- uptake. J. Bacteriol. 180:3799-3803.

66. Jeanjean, R., H. C. P. Matthijs, B. Onana, M. Havaux, and F. Joset. 1993. Exposure of the Cyanobacterium Synechocystis PCC6803 to Salt Stress Induces Concerted Changes in Respiration and Photosynthesis. Plant and Cell Physiology. 34:1073-1079.

67. Feng, S., S. M. Powell, R. Wilson, and J. P. Bowman. 2013. Light-stimulated growth of proteorhodopsin-bearing sea-ice psychrophile Psychroflexus torquis is salinity dependent. Isme J. 7:2206-2213. doi: 10.1038/ismej.2013.97; 10.1038/ismej.2013.97.

68. Horie, Y., Y. Ito, M. Ono, N. Moriwaki, H. Kato, Y. Hamakubo, T. Amano, M. Wachi, M. Shirai, and M. Asayama. 2007. Dark-induced mRNA instability involves RNase E/G-type endoribonuclease cleavage at the AU-box and SD sequences in cyanobacteria. Mol. Genet. Genomics. 278:331-346. doi: 10.1007/s00438-007-0254-9 [doi].

69. Sakurai, I., D. Stazic, M. Eisenhut, E. Vuorio, C. Steglich, W. R. Hess, and E. M. Aro. 2012. Positive regulation of psbA gene expression by cis-encoded antisense RNAs in Synechocystis sp. PCC 6803. Plant Physiol. 160:1000-1010. doi: 10.1104/pp.112.202127 [doi].

70. Herranen, M., E. M. Aro, and T. Tyystjarvi. 2001. Two distinct mechanisms regulate the transcription of photosystem II genes in Synechocystis sp. PCC 6803. Physiol. Plant. 112:531-539. doi: ppl1120410 [pii].

71. Eriksson, J., G. F. Salih, H. Ghebramedhin, and C. Jansson. 2000. Deletion mutagenesis of the 5' psbA2 region in Synechocystis 6803: identification of a putative cis element involved in photoregulation. Mol. Cell Biol. Res. Commun. 3:292-298. doi: 10.1006/mcbr.2000.0227 [doi].

72. Mohamed, A., and C. Jansson. 1991. Photosynthetic electron transport controls degradation but not production of psbA transcripts in the cyanobacterium Synechocystis 6803. Plant Mol. Biol. 16:891-897.

73. Mohamed, A., and C. Jansson. 1989. Influence of light on accumulation of photosynthesisspecific transcripts in the cyanobacterium Synechocystis 6803. Plant Mol. Biol. 13:693-700. 
74. Lindberg, P., S. Park, and A. Melis. 2010. Engineering a platform for photosynthetic isoprene production in cyanobacteria, using Synechocystis as the model organism. Metab. Eng. 12:70-79. doi: 10.1016/j.ymben.2009.10.001; 10.1016/j.ymben.2009.10.001.

75. Salih, G. F., and C. Jansson. 1997. Activation of the silent psbA1 gene in the cyanobacterium Synechocystis sp strain 6803 produces a novel and functional D1 protein. Plant Cell. 9:869-878. doi: 10.1105/tpc.9.6.869 [doi].

76. Ke, N., J. Baudry, T. M. Makris, M. A. Schuler, and S. G. Sligar. 2005. A retinoic acid binding cytochrome P450: CYP120A1 from Synechocystis sp. PCC 6803. Arch. Biochem. Biophys. 436:110120. doi: S0003-9861(05)00007-X [pii].

77. Alder, A., P. Bigler, D. Werck-Reichhart, and S. Al-Babili. 2009. In vitro characterization of Synechocystis CYP120A1 revealed the first nonanimal retinoic acid hydroxylase. Febs J. 276:54165431. doi: 10.1111/j.1742-4658.2009.07224.x.

78. Hoffmann, J., L. Aslimovska, C. Bamann, C. Glaubitz, E. Bamberg, and B. Brutschy. 2010. Studying the stoichiometries of membrane proteins by mass spectrometry: microbial rhodopsins and a potassium ion channel. Phys. Chem. Chem. Phys. 12:3480-3485.

79. Dioumaev, A. K., L. S. Brown, J. Shih, E. N. Spudich, J. L. Spudich, and J. K. Lanyi. 2002. Proton transfers in the photochemical reaction cycle of proteorhodopsin. Biochemistry. 41:5348-5358.

80. Kirchman, D. L., and T. E. Hanson. 2013. Bioenergetics of photoheterotrophic bacteria in the oceans. Environ. Microbiol. Rep. 5:188-199. doi: 10.1111/j.1758-2229.2012.00367.x; 10.1111/j.17582229.2012.00367.x.

81. Lindholm, L., C. Arioz, M. Jawurek, J. Liebau, L. Maler, A. Wieslander, C. von Ballmoos, and A. Barth. 2015. Effect of lipid bilayer properties on the photocycle of green proteorhodopsin. Biochim. Biophys. Acta. 1847:698-708. doi: S0005-2728(15)00069-9 [pii].

82. Branco Dos Santos, F., W. Du, and K. J. Hellingwerf. 2014. Synechocystis: Not Just a Plug-Bug for CO2, but a Green E. coli. Front. Bioeng. Biotechnol. 2:36. doi: 10.3389/fbioe.2014.00036 [doi].

83. Matthijs, H. C., P. M. Visser, B. Reeze, J. Meeuse, P. C. Slot, G. Wijn, R. Talens, and J. Huisman. 2012. Selective suppression of harmful cyanobacteria in an entire lake with hydrogen peroxide. Water Res. 46:1460-1472. doi: 10.1016/j.watres.2011.11.016 [doi].

84. Balashov, S. P., E. S. Imasheva, A. R. Choi, K. H. Jung, S. Liaaen-Jensen, and J. K. Lanyi. 2010. Reconstitution of gloeobacter rhodopsin with echinenone: role of the 4-keto group. Biochemistry. 49:9792-9799. doi: 10.1021/bi1014166.

85. Balashov, S. P., E. S. Imasheva, V. A. Boichenko, J. Anton, J. M. Wang, and J. K. Lanyi. 2005. Xanthorhodopsin: a proton pump with a light-harvesting carotenoid antenna. Science. 309:20612064. doi: 309/5743/2061 [pii].

86. Singh, K., and S. R. Caplan. 1980. The purple membrane and solar energy conversion. Tibs. 5:6264. doi: 10.1016/0968-0004(80)90069-9. 
87. Inoue, K., H. Ono, R. Abe-Yoshizumi, S. Yoshizawa, H. Ito, K. Kogure, and H. Kandori. 2013. A light-driven sodium ion pump in marine bacteria. Nat. Commun. 4:1678. doi: 10.1038/ncomms2689 [doi].

88. Choi, A. R., L. Shi, L. S. Brown, and K. H. Jung. 2014. Cyanobacterial light-driven proton pump, gloeobacter rhodopsin: complementarity between rhodopsin-based energy production and photosynthesis. PLoS One. 9:e110643. doi: 10.1371/journal.pone.0110643 [doi].

89. Varo, G., and J. K. Lanyi. 1991. Thermodynamics and energy coupling in the bacteriorhodopsin photocycle. Biochemistry. 30:5016-5022.

90. Hellingwerf, K. J., W. Crielaard, and H. V. Westerhoff. 1993. Comparison of Retinal-Based and Chlorophyll-Based Photosynthesis: A Biothermokinetic Description of Photochemical Reaction Centers, p. 45-52. In S. Schuster, M. Rigoulet, R. Ouhabi, and J. -. Mazat (eds.), Modern Trends in Biothermokinetics. Springer US.

91. Atsumi, S., W. Higashide, and J. C. Liao. 2009. Direct photosynthetic recycling of carbon dioxide to isobutyraldehyde. Nat. Biotechnol. 27:1177-1180. doi: 10.1038/nbt.1586 [doi].

92. Nakajima, Y., and R. Ueda. 1997. Improvement of photosynthesis in dense microalgal suspension by reduction of light harvesting pigments. J. Appl. Phycol. 9:503-510. doi: 10.1023/A:1007920025419.

93. Melis, A., J. Neidhardt, and J. R. Benemann. 1999. Dunaliella salina (Chlorophyta) with small chlorophyll antenna sizes exhibit higher photosynthetic productivities and photon use efficiencies than normally pigmented cells. J. Appl. Phycol. 10:515-525. doi: 10.1023/A:1008076231267.

94. Erdrich, P., H. Knoop, R. Steuer, and S. Klamt. 2014. Cyanobacterial biofuels: new insights and strain design strategies revealed by computational modeling. Microb. Cell. Fact. 13:128-014-0128-x. doi: 10.1186/s12934-014-0128-x [doi].

95. Groenendijk, G. W., W. J. de Grip, and F. J. Daemen. 1979. Identification and characterization of syn- and anti-isomers of retinaloximes. Anal. Biochem. 99:304-310. 
Table I: Primers used in this study.

\begin{tabular}{|c|c|c|c|}
\hline Name & Fragment & Sequence ${ }^{a}$ & Reference \\
\hline JBS300 & BBa_B0014 & taccctaggaattcgcggccgcttctagagtcacactggctcaccttcg & This study \\
\hline JBS301 & BBa_B0014 & tacctgcagcggccgctactagtaaaataataaaaaagccggattaata & This study \\
\hline JBS302 & $\mathrm{P}_{\text {trc }}-\mathrm{RBS}-\mathrm{Avrll}$ & tacgaattcgcggccgcttctagagttgacaattaatcatccggc & This study \\
\hline JBS303 & $P_{t r c}-R B S-A v r l l$ & tacctgcagcggccgctactagtacctaggtcctccttgtgtgaaattgttatccgc & This study \\
\hline JBS306 & PR-His/PROPS & tacttctagatgaaattattactgatattagg & This study \\
\hline JBS307 & PROPS & tacttctagaattaagcattagaagattctttaac & This study \\
\hline JBS311 & PR-His & tacttctagaattagtgatggtgatggtgatgagcattagaagattctttaac & This study \\
\hline JBS319 & PROPS_1 & $\underline{\text { ctgttagtaaccaattaatgtatctaaatac }}$ & This study \\
\hline JBS320 & PROPS_2 & gtatttagatacattaattggttactaacag & This study \\
\hline JBS339 & $\mathrm{P}_{r n p B}-\mathrm{RBS}-\mathrm{Avrll}$ & tacgaattcgcggccgcttctagagttcaatgcggtccaatacctc & This study \\
\hline JBS340 & $\mathrm{P}_{r n p B}$-RBS-Avrll & ctgcagcggccgctactagtacctaggtcctccttttttctagtgtgccattgattag & This study \\
\hline JBS341 & $\mathrm{P}_{p s b A 2}-\mathrm{RBS}-\mathrm{Avrll}$ & tacgaattcgcggccgcttctagagttgactaattgtatgcccgac & This study \\
\hline JBS342 & $\mathrm{P}_{p s b A 2}-\mathrm{RBS}-\mathrm{Avrll}$ & tacctgcagcggccgctactagtacctaggtcctcctatgtatttgtcgatgttcagatt & This study \\
\hline
\end{tabular}

${ }^{a}$ Sequences that overlap with the sequence of the target fragment are underlined. 


\section{Legends to the figures:}

Figure 1: Expression of His-tagged PR in a batch culture of Synechocystis in various growth phases. Cells were grown in BG-11 medium at moderate light intensities (for further detail: see the text). A) The filled squares show the cell density as monitored via the $\mathrm{OD}_{730}$. At several time points, sample was removed for PR-His quantification via Western blots and the results obtained are expressed as the grey bars (as the weight percentage of PR-His protein over the total protein content of the cell extracts (i.e. $\mu \mathrm{g}$ of PR / $\mu \mathrm{g}$ total protein $\times$ 100). B) The Western blot corresponding to this experiment. Lanes 1-5 contain the samples that represent the grey bars in panel A. Lanes 6-10 contain a decreasing amount of His-tagged PR purified from E. coli. The data shown are from a representative experiment.

Figure 2: Localization of His-tagged $P R$ in the cytoplasmic membrane fraction (CM) and the thylakoid membrane fraction (TM) of Synechocystis. Membranes were separated by two-phase separation and analyzed on Western blots as described in Materials \& Methods. A) Localization of PR in the membrane fractions. B) Localization of PsbC (CP43), a marker for the TM fraction. C) Localization of PixJ1, a marker for the CM fraction. Note that the image shown for each panel was derived from separate gels and blots, which were run under optimized conditions for the particular protein/antibody combination. Because of this, and because of the different antibodies used, the band intensities are not comparable between panels. However, an equal total protein amount of the TM and CM fractions was always loaded on each individual gel (10 $\mu \mathrm{g}, 10 \mu \mathrm{g}$ and $60 \mu \mathrm{g}$ for panels A, B and C, respectively). Thus, the band intensities of both fractions in the same panel can be directly compared. The experiment shown here was repeated with an independently grown culture and independently isolated membrane fractions, and had the same outcome.

Figure 3: Extraction of retinal from Synechocystis that do (pQC006) and do not (pJBS1312) express PR. Retinal standards or cell extracts were incubated with hydroxylamine to allow the formation of retinal oxime, which was subsequently extracted with petroleum ether and detected with HPLC (see Materials \& Methods for further detail). A) Time traces of the absorption at $357.7 \mathrm{~nm}$-the wavelength of maximum absorption of retinal oxime in the system used. The arrow $A$ at $\sim 4.1 \mathrm{~min}$ indicates the maximum absorption of syn-retinal oxime, while the arrow B at $\sim 4.5$ min indicates the peak of anti-retinal oxime (95). B) The absorption spectrum of the maximum of the peak containing retinal oxime from the standard sample (dotted line) and the corresponding peak from the strain carrying pQC006 (solid line). The peak was absent in the strain carrying the empty plasmid pJBS1312 (dashed line). For display purposes, an arbitrary offset was added to the traces of panel $A$, and in panel $B$ the absorption at 357.7 of the retinal oxime standard was normalized to the same absorption at $357.7 \mathrm{~nm}$ as the pQC006 sample.

Figure 4: Isolation and fractionation of holo-PR from Synechocystis. His-tagged holo-PR was isolated using a HisTrap column, and further fractionated and purified using gel filtration chromatography. A) Absorption at $525 \mathrm{~nm}$ of fractions from the gel filtration column. The inset shows the protein as loaded on the gel filtration column. B) Absorption spectra of the two fractions indicated with arrows in panel A (solid line for fraction A; dashed line for fraction B). Spectra were normalized for visual comparison.

Figure 5: Identification of the chromophore via mass spectrometry. The protein was first isolated with its associated chromophore using a HisTrap column, further purified using gel filtration (Figure 4), and finally the chromophore was extracted as retinal oxime with hydroxylamine. Several fractions were then analyzed using 
mass spectrometry. Fraction A is shown here, but results for fraction B were comparable. The bottom panel shows the mirrored spectrum of a retinal oxime standard.

Figure 6: PR-stimulation of the growth of Synechocystis sp. PCC 6803. Comparison of strains with the empty plasmid pJBS1312 (triangles), plasmid pQC006 that leads to expression of PR-His (squares), or plasmid pQC011, expressing the non-proton-pumping PROPS (35) homologue of PR (circles). A) Growth recorded via measurements of $\mathrm{OD}_{750}$. B) Growth analyzed via measurement of the number of cells per ml. Data shown are from a representative experiment from a series of five (A) and two (B) independent experiments. Error bars represent the standard deviation of twelve technical replicates within the representative experiment $(n=12)$, and are only visible when they exceed the size of the symbols. Time point zero indicates the inoculation of the cultures from a pre-culture growing linearly in the same medium. 